\title{
The Question That Doesn't Seem to Go Away: Cancer Risk of Anti-TNF Therapy
}

\author{
Oren Ledder ${ }^{1,2}$
}

Accepted: 19 January 2021 / Published online: 17 February 2021

(c) The Author(s), under exclusive licence to Springer Science+Business Media, LLC part of Springer Nature 2021

Anti-tumor necrosis factor (TNF) therapy has revolutionized the treatment of inflammatory bowel disease (IBD) over the past two decades; for much of this period, the unresolved question of the potential cancer risk of this class of drug has been overshadowed by its therapeutic successes. Pediatriconset IBD per se is associated with an increased risk of cancer overall, primarily of skin, lymphoid, and gastrointestinal origin according to large population studies [1, 2]. In these, the risk estimates were similar regardless of exposure to corticosteroids, thiopurines, and/or anti-TNF therapy.

More specifically, thiopurine therapy for IBD has been associated with an increased lymphoma risk. A recent large meta-analysis by Kotlyar et al. [3] demonstrated a pooled standardized incidence ratio of 4.92 with the risk becoming significant after one year of exposure. Though younger patients had the highest relative risk, particularly young men, the absolute risk remained low in the younger age group with the number of patients needed to treat to cause one additional lymphoma estimated at 4,598 in the 20-29year bracket. Furthermore, the risk normalized following treatment cessation.

While the increased risk of lymphoma with thiopurine treatment has been well established, the contribution of antiTNF therapy to cancer risk has remained more elusive. TNF activates nuclear factor- $\mathrm{\kappa B}$, a mediator of cell apoptosis; hence, TNF blockade may potentially be tumor promoting [4]. Yet the true contribution of anti-TNF therapy to lymphoma risk is confounded by the use of adjuvant immunosuppressive agents in most of these patients. A large nationwide register-based Danish cohort study of IBD patients revealed no significant association between anti-TNF therapy

Oren Ledder

orenl@szmc.org.il

1 Juliet Keidan Department of Paediatric Gastroenterology, Pediatric Gastroenterology and Nutrition Unit, Shaare Zedek Medical Center, P.O.B 3235, 91031 Jerusalem, Israel

2 The Hebrew University of Jerusalem, Jerusalem, Israel and cancer after adjusting for thiopurine use over median follow-up of 3.7 years [5]. Hematopoietic cancers decreased from RR 1.36 (95\% CI 0.67-2.76) overall to 0.90 (95\% CI $0.42-1.91$ ) in the adjusted model, accounting for thiopurine use. Supporting this finding is a pooled analysis of data from multiple clinical trials of adalimumab in which a threefold increased relative risk of malignancy was seen in individuals managed with combination therapy, but with no increased risk in monotherapy [6]. However, median follow-up in this study was limited to $1.25-1.61$ years in these groups. Pediatric-specific data also demonstrate a similar rate of lymphoma among patients treated with anti-TNF agents to the expected background pediatric lymphoma risk [7].

Conflicting with these data is a recent pooled analysis of four observational studies including over 250,000 patients [8]. Patients exposed to anti-TNF monotherapy had a pooled incidence rate ratio of 1.52 (95\% CI 1.06-2.19) compared with patients with IBD unexposed to anti-TNF and thiopurines. While this is lower than the RR of 2.23 (95\% CI 1.79-2.79) and 3.71 (95\% CI 2.30-6.00) for those receiving thiopurine monotherapy and combination therapy, respectively, these data suggest an increased risk of lymphoma in patients receiving anti-TNF monotherapy. Nevertheless, and most recently, a systematic review of 28 observational cohort studies including nearly 300,000 patients with IBD found no association between anti-TNF therapy and malignancies in all but one of the studies analyzed [9].

It is with this backdrop that Llanos-Chea et al. [10] in this issue of Digestive Diseases and Sciences present their case series of lymphoma in pediatric-onset IBD treated with infliximab monotherapy. The five cases presented were identified over a two-year period at three tertiary care centers. The mean age at IBD diagnosis was 14.2 years, and median duration of anti-TNF monotherapy was 4.3 years. Two patients were diagnosed with Hodgkin lymphoma and three with non-Hodgkin lymphoma. Three of these patients had lymphoma involving bone, a feature not previously identified in studies assessing lymphoma risk in IBD. The "per-center" 
risk of lymphoma among those patients on infliximab monotherapy was $3.1 \%$ and $1.5 \%$ as per the research patient data registry and manual chart review, respectively.

Though these cases are an important learning point and of interest to treating gastroenterologists both for their seeming novelty and gravity, care should be taken when extrapolating these cases to clinical practice. While according to several large population studies and systematic reviews, anti-TNF agents are likely not associated with increased malignancy overall, and lymphoma in particular, the last word on this matter is yet to be said. Considering the high per-center risk and the novel nature of the malignancies presented in this case series, should this finding be extrapolated one would have expected some supporting data from existent studies.

Furthermore, as large tertiary referral centers, it would be expected that patients with IBD with lymphoma would be managed in these centers as opposed to smaller IBD units or community centers. Hence, the apparent incidence of lymphoma in IBD in these centers could potentially be skewed due to referral bias, artifactually increasing lymphoma incidence.

Beyond the potential statistical risk of lymphoma associated with anti-TNF therapy, the clinical significance of this is likely minimal. Even in those studies that do demonstrate a statistically significant risk of lymphoma, the relative risk is far below that seen with thiopurines, yet the importance of thiopurines in IBD therapy remains firmly established. In the cost-benefit analysis undertaken by the treating physician prior to any treatment change, on balance with the substantial benefit of anti-TNF therapy in IBD, its importance in clinical practice remains near certain.

\section{Compliance with Ethical Standards}

\author{
Conflict of interest None.
}

\section{References}

1. Olen O, Askling J, Sachs MC et al. Childhood onset inflammatory bowel disease and risk of cancer: a Swedish nationwide cohort study 1964-2014. BMJ 2017;358:j3951

2. Kjaergaard VS, Jensen CB, Elmahdi R, Burisch J, Allin KH, Jess $\mathrm{T}$. Cancer risk in pediatric-onset inflammatory bowel disease: a population-based Danish cohort study. Gastroenterology 2020;159:1609-1611

3. Kotlyar DS, Lewis JD, Beaugerie L et al. Risk of lymphoma in patients with inflammatory bowel disease treated with azathioprine and 6-mercaptopurine: a meta-analysis. Clin Gastroenterol Hepatol 2015;13:847-858 (quiz e48-50).

4. Balkwill F. Tumour necrosis factor and cancer. Nat Rev Cancer 2009;9:361-371

5. Nyboe Andersen N, Pasternak B, Basit S et al. Association between tumor necrosis factor-alpha antagonists and risk of cancer in patients with inflammatory bowel disease. JAMA 2014;311:2406-2413

6. Osterman MT, Sandborn WJ, Colombel JF et al. Increased risk of malignancy with adalimumab combination therapy, compared with monotherapy, for Crohn's disease. Gastroenterology 2014;146:941-949

7. Dulai PS, Thompson KD, Blunt HB, Dubinsky MC, Siegel CA. Risks of serious infection or lymphoma with anti-tumor necrosis factor therapy for pediatric inflammatory bowel disease: a systematic review. Clin Gastroenterol Hepatol 2014;12:1443-1451 (quiz e88-9).

8. Chupin A, Perduca V, Meyer A, Bellanger C, Carbonnel F, Dong C. Systematic review with meta-analysis: comparative risk of lymphoma with anti-tumour necrosis factor agents and/or thiopurines in patients with inflammatory bowel disease. Aliment Pharmacol Ther 2020;52:1289-1297

9. Muller M, D’Amico F, Bonovas S, Danese S, Peyrin-Biroulet L. TNF inhibitors and risk of malignancy in patients with inflammatory bowel diseases: a systematic review. J Crohns Colitis 2020.

10. Llanos-Chea A, Shapiro J, Winter R et al. Lymphoma in pediatriconset inflammatory bowel disease treated with infliximab monotherapy: a case series. Dig Dis Sci. (Epub ahead of print). https:// doi.org/10.1007/s10620-021-06884-9.

Publisher's Note Springer Nature remains neutral with regard to jurisdictional claims in published maps and institutional affiliations. 